\title{
Errors in Device Localization in MRI Using Z-Frames
}

\author{
Jeremy Cepek ${ }^{1,2}$, Blaine A. Chronik ${ }^{1,3}$, and Aaron Fenster ${ }^{1,2}$ \\ ${ }^{1}$ Robarts Research Institute, The University of Western Ontario, London, Canada \\ \{jcepek, afenster\} arobarts.ca, bchronik@uwo.ca \\ ${ }^{2}$ Biomedical Engineering, The University of Western Ontario, London, Canada \\ ${ }^{3}$ Department of Physics and Astronomy, The University of Western Ontario, London, Canada
}

\begin{abstract}
The use of a passive MRI-visible tracking frame is a common method of localizing devices in MRI space for MRI-guided procedures. One of the most common tracking frame designs found in the literature is the z-frame, as it allows six degree-of-freedom pose estimation using only a single image slice. Despite the popularity of this design, it is susceptible to errors in pose estimation due to various image distortion mechanisms in MRI. In this paper, the absolute error in using a z-frame to localize a tool in MRI is quantified over various positions of the zframe relative to the MRI isocenter, and for various levels of static magnetic field inhomogeneity. It was found that the error increases rapidly with distance from the isocenter in both the horizontal and vertical directions, but the error is much less sensitive to position when multiple contiguous slices are used with sliceselect gradient nonlinearity correction enabled, as opposed to the more common approach of only using a single image slice. In addition, the error is found to increase rapidly with an increasing level of static field inhomogeneity, even with the $\mathrm{z}$-frame placed within $10 \mathrm{~cm}$ of the isocenter.
\end{abstract}

Keywords: MRI-guided, stereotactic, z-frame, passive tracking.

\section{Introduction}

\subsection{Background}

The use of MRI for guiding interventional or diagnostic tools into human tissues is becoming increasingly prevalent. The choice of MRI as the guiding imaging modality is often due to its ability to produce high-resolution, high contrast images of soft tissues, and its intrinsic 3D acquisition capability: i.e. the ability to acquire single or contiguous image slices or image volumes in any arbitrary orientation. MRI allows multiple different tissue contrast mechanisms, the combination of which can provide unique information to radiologists for disease detection and grading. If a mechanical or robotic device is to be used for an MRI-guided procedure, a method of mapping coordinates between MRI space and device space is required. This mapping is generally performed by finding a rigid transformation that relates the two coordinate systems.

A common method of estimating the transform between device and MRI space in MRI-guided procedures is with the use of passive MRI-visible tracking frames. Passive tracking frames employ MRI-visible markers arranged in a known geometric 
configuration. Once imaged, a relationship between the device and MRI coordinate systems can be established, allowing any point in MRI space to be targeted by the interventional device. One of the tracking frames most commonly used for device localization in MRI is any one of the variations of designs based on the BrownRoberts-Wells (BRW) frame, initially developed for CT-guided neurosurgical interventions.[1] This frame will hereafter be referred to simply as the 'z-frame'. This frame is attractive for many applications, because a full six degree-of-freedom estimate of a device's pose in MRI coordinates can be obtained using a single image slice of the frame (in principle). However, the nature of geometric distortion in MRI is much different from that in $\mathrm{CT}$, and it is therefore necessary to consider the sources of error in localizing this frame in MRI. Geometric distortion in MRI is highly dependent on the level of magnetic field inhomogeneity (which can be both substantial and unpredictable in the region surrounding a patient's body and near devices containing magnetic materials), as well as the frame's location relative to the scanner's isocenter, which may be unfavorable due to patient positioning.

There are currently several devices in the literature employing the use of a $\mathrm{z}$-frame for registering MRI-guided devices, and several authors have attempted to quantify its performance.[2-6] In [2], DiMaio et al. quantified the tracking accuracy of a z-frame with images acquired at varying slice positions and angles. However, their errors were defined relative to the orientation of the $\mathrm{z}$-frame determined in a baseline image, and therefore give no indication of absolute error in localizing the frame in MRI. They also did not measure localization accuracy at an appreciable distance from the isocenter, which is important for devices with tracking frames positioned external to the patient's body. In [6], Tokuda et al. used a z-frame to register a needle template for MRI-guided transperineal prostate biopsy, and performed tests to quantify the error in $\mathrm{z}$-frame registration; however, they did not consider positioning of the $\mathrm{z}$-frame at a distance from the isocenter along the z-axis (in the LPS patient coordinate system, with the patient positioned head-first supine). Such a configuration is important to consider for applications such as handheld device tracking, as demonstrated by DiMaio et al. [2]. It has also been suggested by Cepek et al. [7] that some clinical MRI scanners may not be able to accurately model the motion of the scanner table, indicating the need to quantify errors in off-isocenter frame localization independently of scanner table motion errors. Tokuda et al. [6] also presented tooltip localization error for a needle lying $100 \mathrm{~mm}$ from the center of the $\mathrm{z}$-frame, but this error would not be sensitive to rotational error in the z-axis. Finally, as was the case in [2], the errors reported in [6] were computed relative to the pose estimate of the z-frame in a baseline MRI scan, and therefore do not quantify absolute error. In [8], Tokuda et al. reported absolute error in localizing targets in a phantom using a z-frame, and found an RMS error of $3.7 \mathrm{~mm}$ for outer targets and $1.8 \mathrm{~mm}$ for inner targets (representative of targets within the prostate capsule). However, their results are only valid for one configuration of the $\mathrm{z}$-frame relative to one specific type of anatomy (prostate). The authors in [4] presented results of needle placement accuracy in MRI using a device employing a z-frame, but these results include other sources of error and cannot be used to predict the error in $\mathrm{z}$-frame registration alone. 
In this work, the absolute error in using a z-frame to localize a tooltip in MRI is quantified for: a) varied position of the $z$-frame relative to the MRI isocenter, and $b$ ) various levels of static field inhomogeneity. In addition, the effect of slice-select error due to gradient field nonlinearity is quantified by imaging the $\mathrm{z}$-frame with either a single image slice or multiple contiguous slices with slice-select gradient nonlinearity correction enabled (hereafter referred to as 3D distortion correction). This is achieved by fixing an MRI-visible spherical marker to a holder of known geometry, onto which a $\mathrm{z}$-frame is mounted. The holder allows the spherical marker to be placed at the MRI isocenter for accurate imaging, and for the position of $\mathrm{z}$-frame to be varied relative to the marker in an accurate manner. The estimated position of the spherical marker, as calculated using the $\mathrm{z}$-frame registration, is compared to its true position, which is measured in tri-planar, high-resolution spin echo images.

\section{Methods}

\subsection{Z-Frame Registration}

The z-frame consists of an arrangement of seven MRI-visible cylinders. A single image slice will show seven ellipses, the centroids of which are used for localizing the frame. The frame is shown in Figure 1.

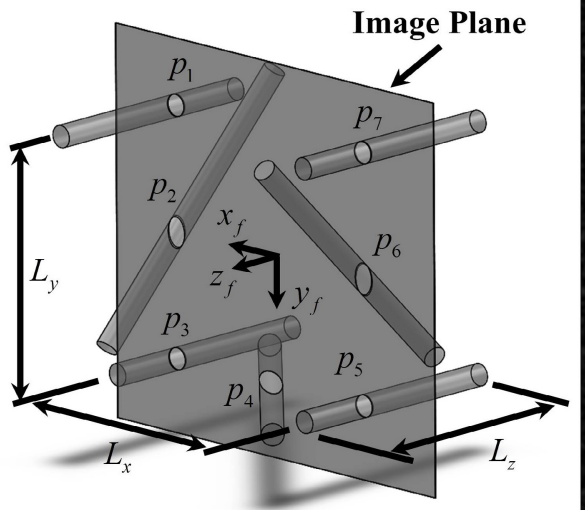

a)

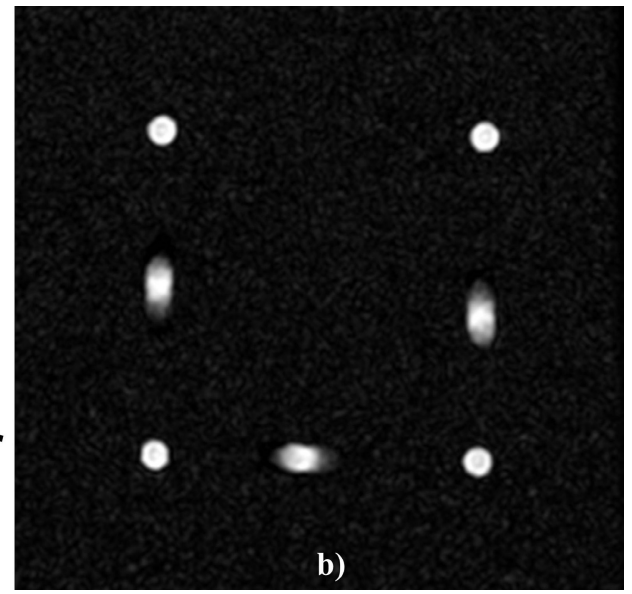

b)

Fig. 1. The z-frame: a) coordinate system and image intersection points, b) MR image showing the seven ellipses

The three corresponding points in the frame coordinate system can be found as

$$
P_{2_{f}}=\left[-\frac{L_{x}}{2}, L_{y}\left(\frac{1}{2}-\frac{d_{23}}{d_{13}}\right), L_{z}\left(-\frac{1}{2}+\frac{d_{23}}{d_{13}}\right)\right],
$$




$$
\begin{gathered}
P_{4_{f}}=\left[L_{x}\left(\frac{1}{2}-\frac{d_{45}}{d_{35}}\right), \frac{L_{y}}{2}, L_{z}\left(-\frac{1}{2}+\frac{d_{45}}{d_{35}}\right)\right], \text { and } \\
P_{6_{f}}=\left[\frac{L_{x}}{2}, L_{y}\left(-\frac{1}{2}+\frac{d_{67}}{d_{57}}\right), L_{z}\left(-\frac{1}{2}+\frac{d_{67}}{d_{57}}\right)\right],
\end{gathered}
$$

where $d_{i j}$ is the Euclidean distance between two points $p_{i}$ and $p_{j}$, which are the centroids of ellipses identified in the MR images. The transformation between these three points and those in MRI coordinates can be used to locate any point known in frame coordinates in MRI coordinates, or vice versa. Due to geometric distortion in the images of the frame, an exact rigid transformation between these points will not generally exist. Thus, a rigid transformation that minimizes the sum of squared distances between corresponding points is sought. Once this rigid transformation is found, any point known in the frame coordinate system can be found in the MRI coordinate system as:

$$
P_{m r}=R_{f} P_{f}+T_{f}
$$

where $P_{m r}$ and $P_{f}$ are the coordinates of a point in the MR and frame coordinate systems, respectively, $R_{f}$ is a rotation matrix, and $T_{f}$ is a translation vector.

\subsection{Experimental Apparatus}

A holder was constructed that allows the spherical marker and $z$-frame to be fixed at precisely-known, discrete distances from each other. Varying the position of the $\mathrm{z}$-frame is possible in both the $\mathrm{y}$ and $\mathrm{z}$ directions over a 3 x 3 grid of positions. The holder is designed so that, in any configuration, the spherical marker can be positioned at the MRI isocenter for accurate localization, and the z-frame can be imaged at a distance from the isocenter. Note that bed movement was not permitted during imaging, so that errors in $\mathrm{z}$-frame localization due to offsets in the $\mathrm{z}$ direction could be quantified. The holder and $z$-frame were constructed from acetyl homopolymer, and the $\mathrm{z}$-frame and spherical marker were filled with a $1 \%$ solution (by volume) of Gd-DTPA (Magnevist, Bayer Healthcare, Berlin, Germany) in distilled water. Localization error is defined as the Euclidean distance between the predicted marker location, computed from the $\mathrm{z}$-frame registration, and its true position as measured in the high-resolution spin echo images. Accordingly, this measure is representative of the error in estimating the location of a tooltip or needle fixed to an interventional device at various distances from the localization frame. The $\mathrm{z}$-frame has a size of $\mathrm{Lx}=\mathrm{Ly}=$ $\mathrm{Lz}=50.8 \mathrm{~mm}$, as defined in Figure 1. The holder is shown in Figure 2. 


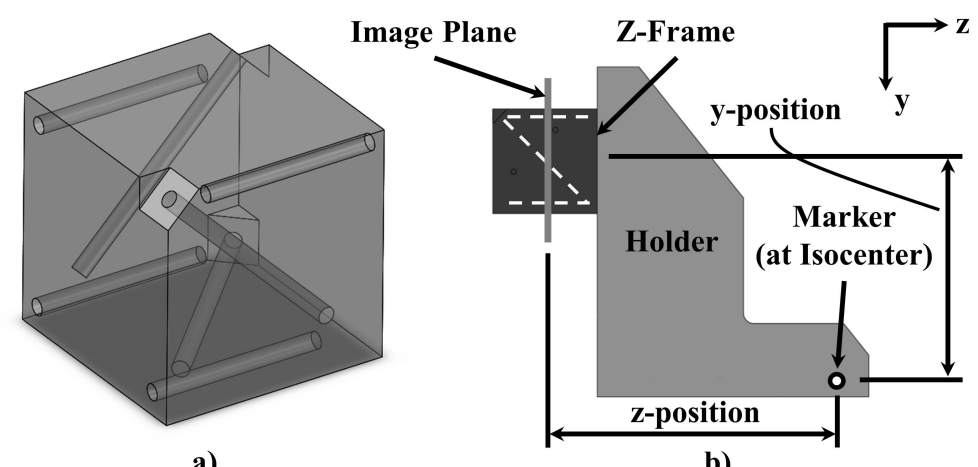

a)

b)

Fig. 2. The z-frame testing apparatus: a) CAD model of the z-frame used for the tests, b) setup of the $\mathrm{z}$-frame and spherical marker on the holder. The spherical marker was positioned within $2 \mathrm{~cm}$ of the isocenter, and the position of the $\mathrm{z}$-frame was varied in the $\mathrm{y}$ and $\mathrm{z}$ directions (in the LPS coordinate system for a patient positioned head-first supine).

\subsection{MRI Acquisitions}

The MRI sequence parameters for imaging of the spherical marker were selected to provide high-resolution images of the marker for accurate localization at the isocenter. Those for the z-frame images were chosen to be typical of the types of scans found in the literature for imaging this type of frame, which are usually tuned for fast image acquisition. The effect of uniform static field inhomogeneity was simulated by varying the scanner's center frequency. All images were acquired in a 3T MRI scanner (MR750, GE Healthcare, Milwaukee, WI). The parameters are shown in Table 1.

Table 1. MRI parameters used for accuracy test

\begin{tabular}{lccc}
\hline \multicolumn{1}{c}{ Parameter } & Spherical Marker & Z-Frame & Units \\
\hline \hline Sequence Type & Spin echo & Fast gradient recalled echo & - \\
\hline Repetition Time & 350 & 150 & $\mathrm{~ms}$ \\
\hline Echo Time & 12 & 4 & $\mathrm{~ms}$ \\
\hline Flip Angle & 90 & 60 & o \\
\hline Bandwidth & 230 & 244 & $\mathrm{~Hz} / \mathrm{pixel}$ \\
\hline Slice Thickness & 1.5 & 3 & $\mathrm{~mm}$ \\
\hline Field-of-view & $60 \times 60$ & $100 \times 100$ & $\mathrm{~mm}$ \\
\hline Acquisition Matrix & $128 \times 128$ & $128 \times 128$ & - \\
\hline$\#$ of Averages & 1 & 1 & - \\
\hline rf coil & Whole body & Whole body & - \\
\hline
\end{tabular}

Centroiding of objects in each image was performed following the method described in [7]. For each z-frame position, four image sets were acquired: single slices with frequency-encoding in both the $\mathrm{x}$ and $\mathrm{y}$ directions, and sets of multiple contiguous slices with $3 \mathrm{D}$ distortion correction enabled and frequency-encoding in both the $\mathrm{x}$ and $\mathrm{y}$ 
directions. Measurements of centroids were only made in the phase-encoded direction of each image. Using this method, which has been described in [7], two images are acquired at the same location, each with the direction of frequency encoding switched. Then, by combining the components of centroids from each image that were taken in the phase-encoded direction, a final in-plane measurement that is insensitive to in-plane distortion due to field inhomogeneity is obtained. The term "distortion correction" refers to a technique whereby a model of the profile of the magnetic field gradients is used to correct distortion in MR images due to gradient field nonlinearity.[9] Most clinical MR scanners feature a factory implementation of such an algorithm. The $2 \mathrm{D}$ version of distortion correction only corrects in-plane distortion, and can therefore be applied to single-slice acquisitions. Correction of out-of-plane distortion can be achieved using a 3D algorithm, but this requires either a 3D acquisition, or an acquisition of multiple contiguous slices. It is important to understand, however, that this method can only correct out-of-plane distortion (slice-select error) caused by gradient nonlinearity; slice-select error due to static field inhomogeneity will still be present. For all images acquired in this work, the factory 2D algorithm was enabled, and the effect of the factory 3D algorithm on localization error was tested.

\section{Results}

Figure 3 shows the error in localizing the spherical marker at different distances along the $y$ - and z-axis of the z-frame from the isocenter. Errors are shown for images acquired with $3 \mathrm{D}$ distortion correction on (using multiple contiguous slices) and 3D distortion correction off (single slice). These measurements are representative of the error in localizing an interventional tool located at the MRI isocenter using a z-frame. Variation in localization error due to image noise was quantified by repeating the experiment five times with the $\mathrm{z}$-frame at its furthest position from the isocenter. At this position, the sensitivity of the localization error to measurement errors is maximized. The standard deviation of error over these five acquisitions was $0.21 \mathrm{~mm}$.

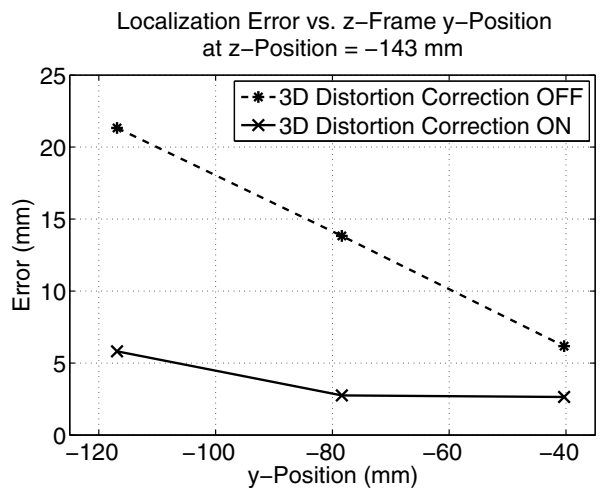

a)

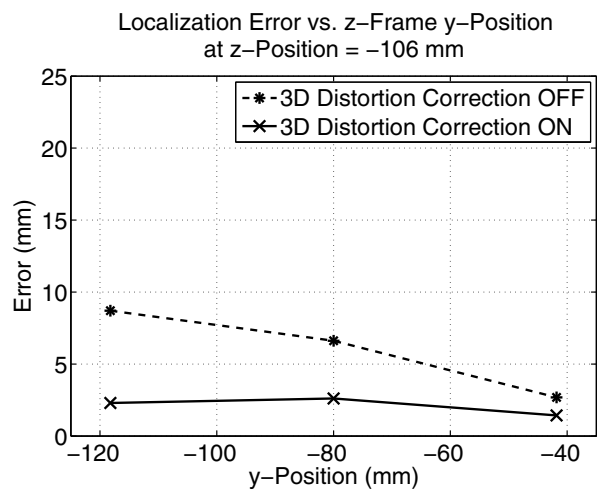

b)

Fig. 3. Tooltip localization error vs. z-frame y-coordinate (relative to isocenter) at three distances from the isocenter along the z-axis. The use of a multi-slice acquisition with $3 \mathrm{D}$ distortion correction greatly reduced the error and its sensitivity to the position of the frame relative to the isocenter. 


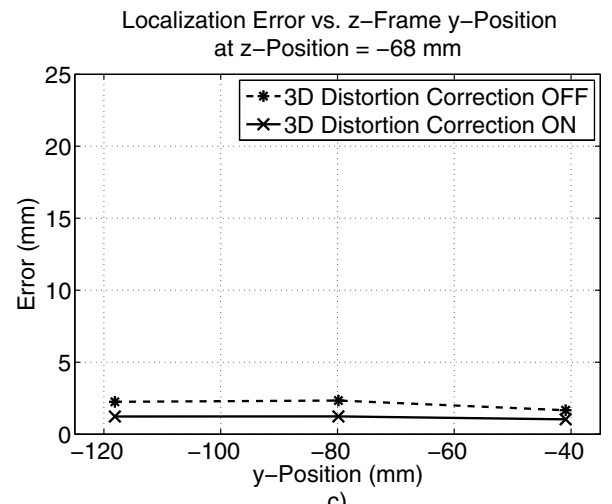

c)

Fig. 3. (continued)

Figure 4 shows the variation in tooltip localization error with increasing static field inhomogeneity with the $\mathrm{z}$-frame at its closest position to the isocenter.

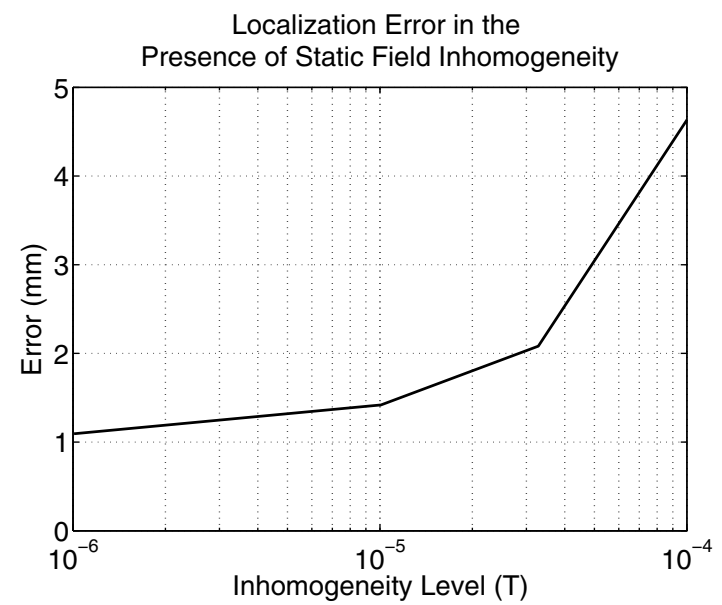

Fig. 4. Tooltip localization error vs. magnitude of static field inhomogeneity with the z-frame located at $\mathrm{x}=0, \mathrm{y}=-40 \mathrm{~mm}, \mathrm{z}=-70 \mathrm{~mm}$ and the spherical marker at the isocenter

\section{Summary}

This work investigated the error in using a $\mathrm{z}$-frame to localize the tooltip of an MRIguided device. It was found that, when using a single image slice, the error increased rapidly with $\mathrm{z}$-frame displacement from the isocenter. However, the use of multiple contiguous slices with 3D distortion correction enabled greatly reduced the dependence of the error on distance from the isocenter, reducing the error to less than $3 \mathrm{~mm}$ in most cases. It was also found that there is a strong dependence of error on static field inhomogeneity, even with the z-frame within $10 \mathrm{~cm}$ of the isocenter. This is 
likely due to the fact that the $\mathrm{z}$-frame pose estimate is sensitive to slice-select error - a major downfall of this frame's design. It is important to note that variation of the $\mathrm{z}$ frame's position strictly along the x-axis is expected to have the same effect as that along the $y$-axis, due to the expected symmetry of gradient hardware and the static field. However, the effect of a combined translation in the $x-y$ plane cannot be extracted from this work. The results of this work will assist anyone developing MRIguided devices to understand the effects of $\mathrm{z}$-frame location, static field inhomogeneity, and 3D distortion correction on device localization accuracy.

\section{References}

1. Brown, R.A.: A stereotactic head frame for use with CT body scanners. Invest. Radiol. 14, 300 (1979)

2. DiMaio, S., Samset, E., Fischer, G., Iordachita, I., Fichtinger, G., Jolesz, F., Tempany, C.: Dynamic MRI scan plane control for passive tracking of instruments and devices. In: Ayache, N., Ourselin, S., Maeder, A. (eds.) MICCAI 2007, Part II. LNCS, vol. 4792, pp. 50-58. Springer, Heidelberg (2007)

3. Fischer, G.S., DiMaio, S.P., Iordachita, I.I., Fichtinger, G.: Robotic assistant for transperineal prostate interventions in 3T closed MRI. In: Ayache, N., Ourselin, S., Maeder, A. (eds.) MICCAI 2007, Part I. LNCS, vol. 4791, pp. 425-433. Springer, Heidelberg (2007)

4. Song, S., Tokuda, J., Tuncali, K., Tempany, C., Zhang, E., Hata, N.: Development and Preliminary Evaluation of a Motorized Needle Guide Template for MRI-guided Targeted Prostate Biopsy. IEEE Trans. Biomed. Eng. (2013)

5. Su, H., Cardona, D.C., Shang, W., Camilo, A., Cole, G.A., Rucker, D.C., Webster, R., Fischer, G.S.: A MRI-guided concentric tube continuum robot with piezoelectric actuation: A feasibility study. In: IEEE International Conference on Robotics and Automation (ICRA), pp. 1939-1945 (2012)

6. Tokuda, J., Tuncali, K., Iordachita, I., Song, S.-E., Fedorov, A., Oguro, S., Lasso, A., Fennessy, F.M., Tempany, C.M., Hata, N.: In-bore setup and software for 3T MRI-guided transperineal prostate biopsy. Phys. Med. Biol. 57, 5823 (2012)

7. Cepek, J., Chronik, B., Lindner, U., Trachtenberg, J., Davidson, S., Bax, J., Fenster, A.: A system for MRI-guided transperineal delivery of needles to the prostate for focal therapy. Med. Phys. 40, 012304 (2013)

8. Tokuda, J., Fischer, G.S., DiMaio, S.P., Gobbi, D.G., Csoma, C., Mewes, P.W., Fichtinger, G., Tempany, C.M., Hata, N.: Integrated navigation and control software system for MRIguided robotic prostate interventions. Computerized Medical Imaging and Graphics: the Official Journal of the Computerized Medical Imaging Society (2009)

9. Wang, D., Strugnell, W., Cowin, G., Doddrell, D.M., Slaughter, R.: Geometric distortion in clinical MRI systems: Part I: evaluation using a 3D phantom. Magn. Reson. Imaging 22, 1211-1221 (2004) 